\title{
A Cervical Smear Scare due to Monsel's Solution: A Case Report
}

\author{
Muhammad Fairuz Abdul Rahman ${ }^{\mathrm{a}, \mathrm{d}}$, Wai Loong Wong ${ }^{\mathrm{b}}$, \\ Sung Hock Chew ${ }^{\mathrm{c}}$, Yen Ching $\mathrm{Yeo}^{\mathrm{c}}$
}

\begin{abstract}
Monsel's solution has been in use since the end of the 19th century for its valuable property of creating hemostasis in minor disruptions of the epithelium. Its styptic effect was first described by Leon Monsel, a pharmacist in the French army, in a letter dated on October 13, 1852. Containing ferric subsulphate, it is used as a topical hemostatic agent in minor procedures such as biopsies in gynecology, proctology, dermatology, otorhinolaryngology and odontology. A 28-yearold nulliparous woman, with a known history of adenomyosis, had presented for sudden heavy intermenstrual vaginal bleeding. Examination revealed a bleeding Nabothian cyst. Monsel's solution application helped stop the bleeding. She returned 3 days later with persistent heavy vaginal bleeding. Examination showed active oozing from the Nabothian cyst. A cervical smear and a human papillomavirus (HPV) swab were taken, and vaginal packing was inserted. The cervical smear result was reported as atypical cells, suspicious for malignancy. Colposcopy performed showed a grade 2 acetowhite lesion, suspicious for cervical intraepithelial neoplasia (CIN) III/microinvasive disease. However, punch biopsies of the cervix revealed only CIN I/ koilocytosis. The patient was counselled and subsequently underwent a laser cone biopsy, which showed CIN I with clear margins. The discrepant results between the cervical smear and the cone biopsy prompted a review of the cervical smear and cervical histology; and a relook at the chronology of events. The cytological features observed in the initial cervical smear could be explained by the interference from the Monsel's solution, which was applied just 3 days before the cervical smear. In cases where Monsel's solution has been used for hemostasis, it is best to delay taking a cervical smear or a biopsy for at least 3 weeks as the histologic effect of Monsel's solution may persist
\end{abstract}

Manuscript submitted July 29, 2020, accepted August 11, 2020

Published online August 28, 2020

aDepartment of Obstetrics and Gynecology, KK Women's and Children's Hospital, Singapore, Singapore

${ }^{b}$ Department of Gynecological Oncology, KK Women's and Children's Hospital, Singapore, Singapore

'Department of Pathology and Laboratory Medicine, KK Women's and Children's Hospital, Singapore, Singapore

${ }^{\mathrm{d} C}$ Corresponding Author: Muhammad Fairuz Abdul Rahman, Department of Obstetrics and Gynecology, KK Women's and Children's Hospital, 100 Bukit Timah Rd, Singapore, Singapore. Email: muhammadfairuzb.abdulrahman@ mohh.com.sg

doi: https://doi.org/10.14740/jmc3556 for up to that duration of time in tissues.

Keywords: Monsel's solution; Cervical smear; Human papillomavirus

\section{Introduction}

Monsel's solution ( $20 \%$ aqueous ferric subsulphate) has been in use in medicine for decades for its styptic qualities to secure hemostasis. It is popular amongst gynecologists performing cervical biopsies and excisions, as well as dermatologists performing punch biopsies. Leon Monsel, a French pharmacist, first described the styptic qualities of ferrous subsulphate in 1852 during the Crimean War [1]. Monsel's solution was initially used for disruptions of the epithelium, which were numerous during wartime [2]. Ferric subsulphate was then compared to ferrous chloride for hemostasis in a study published in the Archives of Dermatology. The authors concluded that its hemostatic effect was due to mechanical sealing of small vessels, rather than the acidic background. By reacting ferric sulphate with sulphuric acid and nitric acid, with a $\mathrm{pH}$ approaching 1, the brown acidic Monsel's solution is created [3]. Its use as a hemostatic agent has been explored beyond cervical biopsies and excision procedures in gynecology. Monsel's solution has been proposed as a treatment option for obstetric hemorrhage, where it is applied directly to the placental bed in a case of focal placenta accreta [4]. However, one has to be re-

\section{Key Points}

- Monsel's solution is an effective topical hemostatic agent for cervical biopsies and excision procedures, which can eliminate the need for suturing.

- The cytological and histological effects of Monsel's solution may persist for up to 3 weeks in tissue.

- It is prudent to wait for at least 3 weeks after the application of Monsel's solution, before a cervical smear or biopsy is taken to avoid diagnostic error and difficulty.

- It is important to highlight to pathology colleagues any unusual sequence of events prior to the collection of a cervical smear or tissue biopsy, in particular if there has been any usage of topical agents like Monsel's solution or other substances that can cause chemical artifacts. 


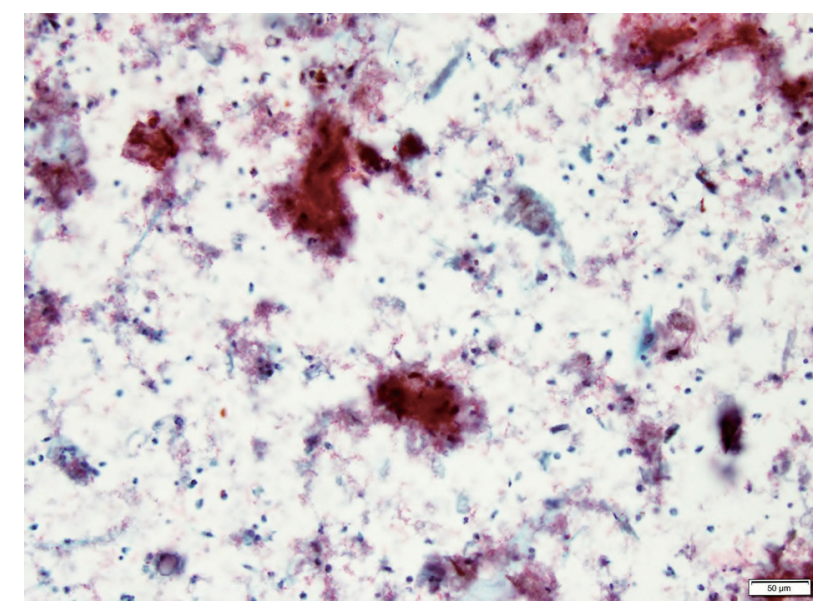

Figure 1. Cervical smear showing a background of hemorrhagic diathesis.

ally careful when examining a cytology or histology specimen that has had a recent "interference" from Monsel's solution, as failure to recognize its related artifact may lead to diagnostic problems, in particular overcalling of cervical smear resulting in false positive diagnosis of a cervical malignancy.

\section{Case Report}

A 28-year-old nulliparous woman with a history of adenomyosis presented to the Urgent Obstetrics and Gynecology Clinic (UOGC) for sudden heavy intermenstrual bleeding. The bleeding had begun as a mild brown vaginal discharge 7 days earlier, which had now become acutely heavy. She had completed her menses, which lasted 3 days, just 12 days prior. She has never had intermenstrual bleeding before. There was no provoking trauma or recent sexual intercourse.

Pelvic examination revealed a cervix with a Nabothian cyst at the posterior lip, which was actively oozing. The uterus felt bulky and adenomyotic, and the cervix felt smooth. Monsel's solution, along with compression, was applied onto the cervix, and the bleeding stopped.

She returned 3 days later for a review. The heavy vaginal bleeding had recurred since a day earlier. Pelvic examination showed active oozing from a $2-\mathrm{cm}$ Nabothian cyst in the posterior cervix, which was otherwise smooth. A cervical smear and a human papillomavirus (HPV) swab were taken. Vaginal packing was inserted, which stopped the bleeding.

Although the HPV test was negative, the cervical smear result showed atypical cells, suspicious for malignancy. In particular, the smear showed a hemorrhagic diathesis (Fig. 1), in which scattered atypical keratinized cells were noted (Fig. 2), leading to a suspicion for malignancy. She was referred for colposcopy.

Colposcopy was adequate with a type 1 transformation zone. A grade 2 acetowhite lesion and a nodule were seen in the posterior cervix, but with no mosaicism, punctation, or atypical vessels. Clinical impression was that of possible cervical intraepithelial neoplasia (CIN) III/microinvasive disease. Punch biopsies taken showed CIN I and koilocytosis. p16

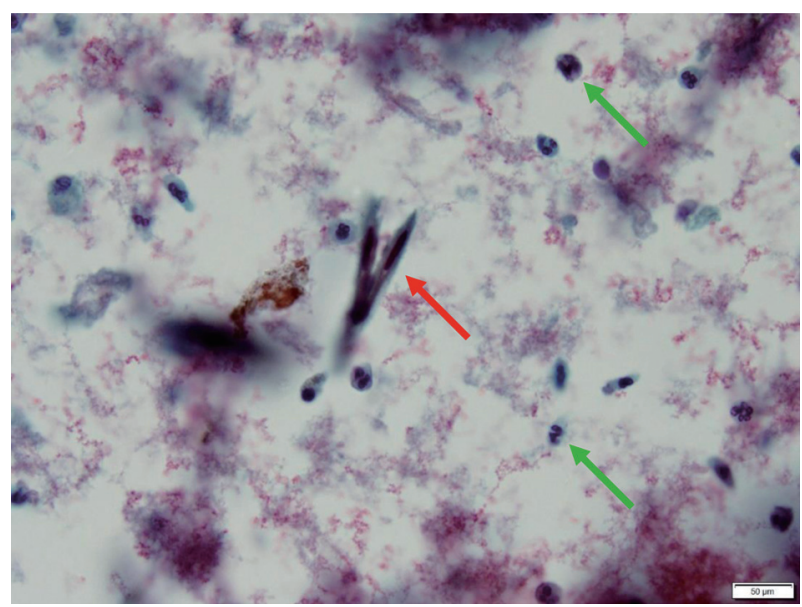

Figure 2. Occasional spindled squamous cells with hyperchromatic nuclei (red arrow) are seen resembling keratinized squamous cells commonly seen in association with invasive squamous cell carcinoma. Polymorphonuclear cells with enlarged nuclei, conspicuous nucleoli and coarse chromatin (green arrows) are seen in the background.

block positivity was not demonstrated. The patient was counselled regarding the discrepancy in results and agreed for a laser cone biopsy of the cervix to exclude malignancy.

During the laser cone biopsy, multiple deep Nabothian cysts were noted at the 8 to 10 o'clock positions. A $15 \times 15$ $\times 12 \mathrm{~mm}$ cone biopsy specimen was obtained. The final histology showed CIN I with clear margins. There was no cervical glandular intraepithelial neoplasia (CGIN) or invasive malignancy. A repeat smear 3 months after the cone biopsy showed atypical squamous cells of undetermined significance (ASCUS) and the repeat HPV test was negative.

The patient made a full recovery from the laser cone biopsy and had a levonorgestrel intrauterine system (LNG-IUS) inserted to reduce her heavy menstrual bleeding due to adenomyosis.

The discrepancy between the initial cervical smear and the final laser cone histology prompted a multidisciplinary discussion involving the gynecologist and the pathologist during which the patient's history and chronology of events were reviewed. It was discovered that Monsel's solution had been applied to the cervix to stop the Nabothian cyst from bleeding, just 3 days before the cervical smear was taken. The conclusion was that Monsel's solution had caused the hemorrhagic diathesis and artifactual changes to squamous cells (Fig. 2), which mimicked the appearance of a cervical squamous cell carcinoma. The patient had even contemplated a hysterectomy but was relieved to know that the discrepant results were due to Monsel's solution-related artifact.

\section{Discussion}

Monsel's solution is an effective topical hemostatic agent, especially ideal for stopping small amounts of bleeding of the cervix or epithelium. Its ability to achieve hemostasis in less than 20 seconds has ensured its wide use in gynecology [5]. Monsel's so- 
lution causes tissue necrosis and impedes re-epithelialization. In the healing phase, granulation tissue, siderophages and ferrugination (i.e., the deposition of ferric salts within fibrin, dermal collagen and striated muscle) are present. Occasionally, a foreign body type reaction to the pigment and necrotic tissue may be seen.

The histologic effect of Monsel's may persist for at least 3 weeks in tissue; hence this may interfere with the ability to recognize and characterize the disease process [6]. If a cervical smear is taken within 3 weeks after the application of Monsel's solution, the changes described above will be reflected in the smear: tumor/hemorrhagic diathesis, spindling of squamous cells and atypical reactive squamous cells showing enlarged nuclei with conspicuous nucleoli (Fig. 2). These cytological features may lead to overcalling of the smear resulting in a false positive diagnosis of invasive squamous cell carcinoma.

Knowing the above, it is prudent to avoid performing a cervical smear on a patient who had just recently been treated with Monsel's solution on the cervix, to avoid confusion in interpreting the cytology. In cases where an urgent smear or biopsy has been taken within a few weeks after Monsel's solution application, this must be highlighted to the pathologist to avoid misinterpretation of the cytologic or histologic findings. Close communication between gynecologist and pathologist is key to avoid misinterpretation of cytology or histology specimens, so as to avoid causing undue anxiety to the patient.

\section{Acknowledgments}

None to declare.

\section{Financial Disclosure}

None to declare.

\section{Conflict of Interest}

None to declare.

\section{Informed Consent}

Informed consent was obtained from the patient.

\section{Author Contributions}

MFAR is involved in data collection, literature review and case write-up. WWL and CSH are involved in supervision and final editing. YYC is involved in supervision, images editing and final editing.

\section{Data Availability}

The data supporting the findings of this study are available from the corresponding author upon reasonable request.

\section{References}

1. Proctor W. Remarks on Monsel's persulphate of iron. Am J Pharmacol. 1859;31:403-407.

2. Garrett AP, Wenham RM, Sheets EE. Monsel's solution: a brief history. J Low Genit Tract Dis. 2002;6(4):225227.

3. Amazon K, Robinson MJ, Rywlin AM. Ferrugination caused by Monsel's solution. Clinical observations and experimentations. Am J Dermatopathol. 1980;2(3):197205.

4. Miller DT, Roque DM, Santin AD. Use of Monsel solution to treat obstetrical hemorrhage: a review and comparison to other topical hemostatic agents. Am J Obstet Gynecol. 2015;212(6):725-735.

5. Capacho A, Alcobia A. PP-004 compounded Monsel's agent in gynaecology procedures. Eur J Hosp Pharm. 2014;21:A123-A124.

6. Spitzer M, Chernys AE. Monsel's solution-induced artifact in the uterine cervix. Am J Obstet Gynecol. 1996;175(5):1204-1207. 